\title{
Why should we Build Artificial Worms and How?
}

\author{
Oded Maler \\ VERIMAG*, Montbonnot, France \\ E-mail: Oded.Maler@imag.fr \\ 1990-1992
}

\begin{abstract}
In this paper it is argued that a certain class of intelligent systems is worth being investigated and developed. This class consists of elastic robots composed of many components having relatively-simple sensory-motor capabilities, whose most prominent biological realizations are worms, molluscs, elephant trunks and tongues. Understanding how such mechanisms are controlled is an important stage (not to say, a prerequisite) toward the development of "truely-intelligent" autonomous agents. As a preliminary step in this direction I describe a simple elastic object considered as a first approximation of an artificial worm and discuss its control principles.

"Worms have played a more important part in the history of the world then most persons would at first suppose." CHARLES DARWIN, 1898.
\end{abstract}

${ }^{*}$ The opinions expressed in this paper do not reflect the policy or scientific interests of any institute to which the author belongs (or belonged in the past). 


\section{Introduction}

In this research we investigate the control principles for a simple elastic object considered as a first approximation of an artificial worm. This work is motivated by the belief that the design of elastic robots composed of many components having relatively-simple sensory-motor capabilities is an important stage in the development of "truly-intelligent" autonomous agents in general, and more flexible robots in particular. The paper is organized as follows. In section 2 we put forward the case for the development of artificial worm-like mechanisms. The current simplified worm model is described in section 3, and the first lessons learnt from our simulation "experiments" are reported in section 4 . I conclude with a brief survey of some relevant and irrelevant work in Robotics, Simulation and Biological modeling. A more detailed description of the extensions of this model (multi-segment and multi-dimensional structures) and a summary of the experiments performed so far will appear in a companion report [MADL92]. More technical details concerning the simulation system appeared in [MAD91].

\section{Motivation and Justification}

It is always worthwhile to remind ourselves what we want to do. Our engineering goal is to develop useful artifacts that can operate in complex and unstructured environments. Our inspiration for the kind of tasks we want these artifacts to perform can come either from the analysis of our needs or through observation of the performance of natural agents of various degrees of complexity. This leads us to the intersection with a parallel scientific goal: building explanatory models of the structure and behavior of existing natural organisms.

The limitations of the so-called traditional AI, inspired by the higher-level capabilities of human beings, are now widely recognized. The ultimate goal of this "symbolic" approach was an artifact capable of passing the Turing test, that is, being able to communicate in natural language. The recent revival of cybernetic models is based on the conviction that the problems associated with the interaction with the concrete, physical world are the important and fundamental ones. Consequently, less-evolved creatures such as insects are taken as normative models for artificial creatures (see [Bro86, MG90, Wi190]). 
The major methodological advantage of this approach (either in a "real" or a simulated world) is that instead of working with abstract rules of the form if the situation is $S$ then perform action $A$, one has to think seriously what it is like for the robot to know that the situation is $S$, given its repertoire of sensors, and what does it mean for it to perform $A$. When considering these kinds of questions (e.g., how can a sonar-based mobile robot know the direction of a corridor or follow a wall) the mistake of taking our own lower-level capabilities for granted is eliminated. This way, folk-psychological over-personification of our own selves is replaced by mechanistic explanations.

Although this trend of focusing on lower-level competence ${ }^{1}$ is favorable, I think that we should get even lower. In other words, insects are far too complex (or intelligent, if you like) animals to begin with. Fortunately, I do not suggest to move the focus of attention down to a quantum-mechanical, nor to a molecular and not even to Protozoan level: I think that the worm ${ }^{2}$ level is the proper one.

The argument I suggest in favor of the worm (with respect to higher-level creatures) is not necessarily based on evolutionary precedence, although this fact can serve as a supporting evidence. Instead it is based on an intuition concerning the computational complexity of sensor-effector loops. As an illustrative examples, let us consider the following control problem: someone is blocking our way and we want to get him away. The most abstract way to achieve this goal is by saying: "would you be so kind as to move". In order for the obstacle to move it needs all the speech recognition apparatus, together with the capability of understanding, a capability which is still mysterious and unexplainable. An alternative approach to the problem might be to wave our hand or to shout loudly in such a way that it will move. The success of this approach depends upon some less sophisticated sensory processing, and it can work even if the obstacle is, say, a dog. The most extremely non-abstract way to achieve this goal is simply to push the obstacle. It will work with any object, either dead or alive, obeying the laws of Newtonian mechanics given that we apply a sufficient amount of force. Suppose now, that we push the obstacle more gently, not strongly enough for making an equivalent mass move, but sufficient enough to cause the object to move.

\footnotetext{
${ }^{1}$ With or without hoping to ever climb up again to the cognitive realm..

${ }^{2}$ From our simplifying point of view, to be described in the sequel, there is no need to substitute the laymen expression "worm" by more refined zoological terms such as Platyhelmints, Nemertines, Nematodes and Annelids (see [FG76]).
} 
For this to occur, the object should have some simple sensors and a simple control mechanism linking its sensors to its locomotion machinery.

The essence of this very informal argument is that tactile sensing (and its associated feed-back loop) is much more concrete than vision (not to mention language). It lies in the boundary between a merely-physical interaction and sensory-based behavior, whereas vision is much more abstract. A pixel on the retina can represent a point on one's nose or another nearby location which is immediately reachable, but it can be reflected as well from a virtually inaccessible mountain top. On the other hand, mechanical sensors are arranged around body boundaries and there is a "smoother" correspondence between the objects we sense and the objects we can influence. I believe that this simple type of relation between perception and action is a fundamental phenomenon that should be investigated before more complex sensor-actuator loops are considered. (Note that it is not an argument against simpler forms of light sensitivity, such as those underlying Phototaxis, but rather against ${ }^{3}$ complex visuomotor coordination tasks as exhibited by insects and higherlevel animals.

One of the most amazing examples of an elastic mechanical organ that does not use vision at all in its feed-back loop is our tongue. Its behavior in controlling our mouth is hidden from our eyes and, how ironically, can hardly be described linguistically ${ }^{4}$ (the reader may try to give a verbal account of what his or her tongue is doing while eating something or exploring a change in the teeth configuration). This is the kind of behavior achievable by elastic mechanisms consisting of rather uniform arrays of simple local sensors and effectors. The worm, a relatively-simple creature with clearly differentiated neuronal and muscular cells, is another example of such a mechanism with a more "autonomous" flavor. As such, it has the methodological advantage of not being partially controlled by a higher-level "conscious" component. 5

The same kind of complexity arguments applies as well the actuator aspects of the loop. The dynamics of limb-based locomotion is much more complex and its control requires much more informational and computational

\footnotetext{
"In fact, it is not an argument "against" anything. I don't consider the investigation of more complex sensory modalities as unimportant but as a very difficult task. All our claims are in the context of methodological efficiency.

${ }^{4}$ Another instance of self-reference?

${ }^{5}$ The internal organs of our body constitute another family of autonomous systems controlled by non-visual signals.
} 
resources than the control of crawling. Again, I speculate that the fundamental principles to be discovered by investigating worms will be useful later for the control of large ensembles of interconnected fibers such as muscles, and that the understanding of these principles will be useful for tackling the additional problems caused by the discontinuities introduced by rigid joints.

After hopefully convincing the reader that the worm is the current upperbound for a successful modeling and imitation effort, one might expect that we prove that it is a lower-bound as well. I hope that the eventual success of this project will constitute a constructive proof. Unlike lower-level animals and substances, many aspects of worm behavior are intuitively comprehensible to a human observer. In the context of robotics, these behaviors are of a similar scale (size and velocity) as the locomotion and manipulation tasks we are interested in. This does not exclude the importance of more fine-grained chemical mechanisms in the future (especially in the implementation of muscle-like contractile fibres), but their investigation requires quite different principles.

\section{What is it Like to Be an Artificial Worm?}

In this section I describe the worm model that we currently use and its natural history as a creation of a non-omnipotent designer. Looking at biological worms we have to decide which of their numerous properties we are going to abstract away. The main characteristic of our artificial worm is not being alive, that is, it is not composed of living cells and it is not self-reproducing. It may turn out that by this decision we miss the essence, and all our effort to achieve "motivation-less" motoric competence are doomed to fail. In this case we might conclude the the causal powers of the cells (to rephrase Searle) are necessary in order to produce the adaptive behavior we are interested in. I hope that this is not the case. It may also turn out that in order to be economic, our worm should adopt some of the peculiar metabolic habits of living organisms, but we are still far from the stage where these considerations should be taken into account.

By getting rid of both food and sex, all that remains is purposeless locomotion. At this level of abstraction our worm is nothing but an articulated mesh of springs lying on some surface. At each time instant it is able to exert some contraction/extraction forces on each of its segments according 
to some control program. This sequence of force applications generates certain movements based on the mechanical properties of the springs and of the parameters determining their interaction with the surface. Our initial aim is to find a control strategy that will produce a "crawling" behavior across a wide variety of environments, such that an artificial worm equipped with this strategy will pass some Turing-like test for worms.

An important methodological consideration is associated with the following question: what does a worm know? We use the term "knowledge" not in the AI sense of an interpreted symbolic structure, but in the sense of a relation between external and internal states (see [Ros 85$]$ ). Thus, the above question is concerned with those physical aspects of the external environment and of its own body that a worm can perceive. Similar questions have been asked in Neurobiology (e.g., [Ste82]) concerning the information measured by various receptors attached to muscles and tendons. It is commonly agreed upon that the relative change in the length of the muscle as well as its derivative are measured by the nervous system and participate in the feed-back loop. From the engineering point of view this question can be rephrased as a controllability/observability problem: which sets measurements and actionss are necessary and sufficient in order to implement an efficient and robust controller for such a device? Since currently we start with uni-directional locomotion along a one-dimensional uniform and unbounded space, we do not need any information beyond the change of length. As the model evolves we will need additional types of "sensors" in order to simulate attraction toward light or food, detection of obstacles, etc. Our guiding principle is to try to achieve as much mechanical performance as possible, relying on the least sufficient information source. Sometimes the notion of minimal information may depend on whether the worm is supposed to be a reasonable biological model or a prototype of a future artifact. Whenever such a conflict occurs we prefer the creative freedom of the (theoretical) engineer over faithfulness to the accidental details associated with existing animals.

In order to demonstrate our approach we will describe the simplest model consisting of a single segment and a single set of control variables. Only after obtaining a clear understanding of the essential principles governing the behavior and control of this basic building block, the control problems associated with an ensemble of interconnected units can be tackled.

The simple mechanical model we employ is the following. The worm is made of two identical masses interconnected by a spring and having corre- 
sponding interaction parameters ${ }^{6}$ with the ground (see figure 1 ). The force $F$ applied on the masses can be either positive (causing expansion) or negative (causing contraction).

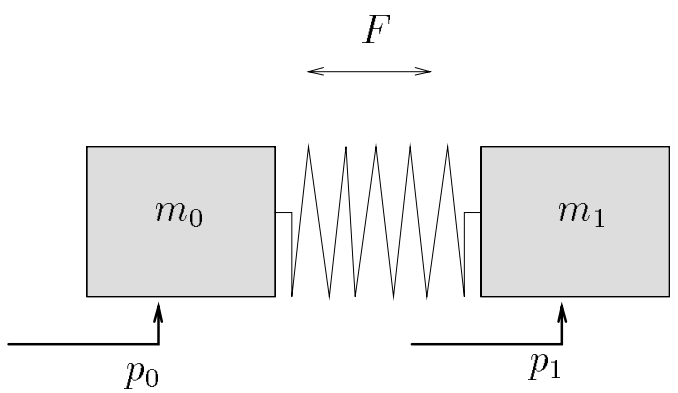

Figure 1: A mechanical model for a one-segment worm.

The overall dynamics is described by the following set of equations:

$$
\begin{aligned}
& +F-k\left(l-l_{r}\right)-\nu \frac{d l}{d t}-m_{0} \frac{d^{2} x_{0}}{d t^{2}}-p_{0} \frac{d x_{0}}{d t}=0 \\
& -F+k\left(l-l_{r}\right)+\nu \frac{d l}{d t}-m_{1} \frac{d^{2} x_{1}}{d t^{2}}-p_{1} \frac{d x_{1}}{d t}=0
\end{aligned}
$$

$\begin{array}{ll}\text { Where } & \begin{array}{ll}m_{0}, m_{1} & \text { are the masses } \\ x_{0}, x_{1} & \text { are their corresponding locations } \\ p_{0}, p_{1} & \text { are the "friction" control parameter for the masses } \\ l_{r} & \text { is the initial rest length of the spring } \\ l=x_{0}-x_{1} & \text { is the actual length of the spring } \\ k & \text { is the stiffness of the spring } \\ \nu & \text { is the damping coefficient of the spring } \\ F & \text { is the applied force }\end{array}\end{array}$

${ }^{6}$ Due to technical reasons, the friction is simulated by two additional constraints attaching the two masses to the ground. They act as dampers with very large coefficients $p_{0}$ and $p_{1}$. 


\section{Preliminary Results}

In this section I describe the experimentation I made with the elementary building-block and the control rule that makes it crawl.

All our experimental models are created and tested using the simulation generation system described in [ADH89] and [ADH91]. This system takes as its input the geometrical and the mechanical description of the worm, performs simplifications using symbolic computations and finally produces a discrete-time simulator based on a numerical resolution procedure. (Some additional worm-related implementation details appear in [MAD91]). The controller is just a simple module (see figure 2) within the simulator which computes $F, p_{0}$ and $p_{1}$ as a function of the current and previous values of $x_{0}$ and $x_{1}$.

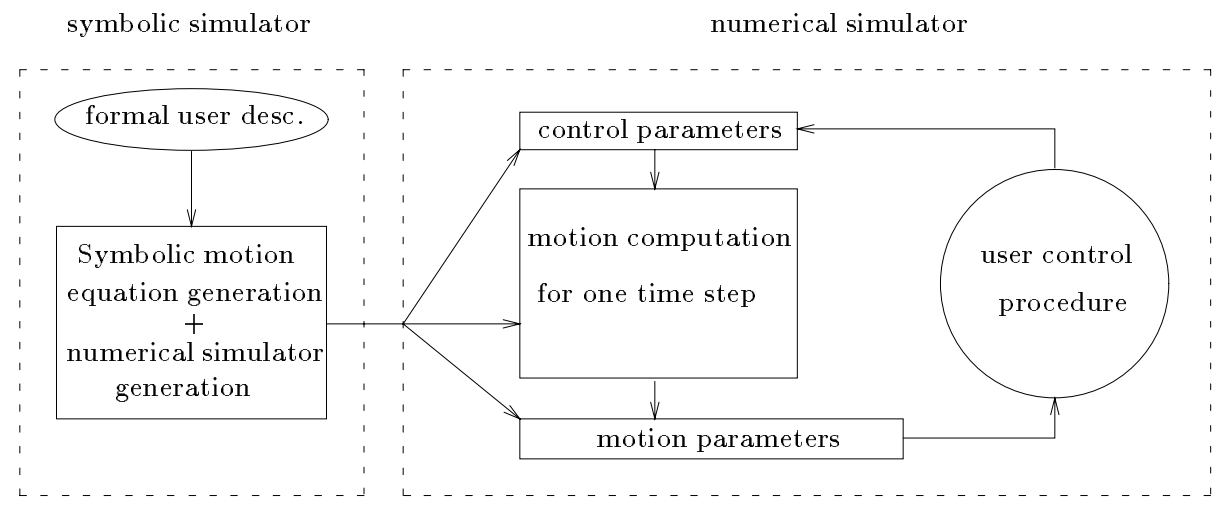

Figure 2: A generic description of the simulation system.

Although this setting allows the worm to control both $F$ and the "friction" coefficients, in the initial model I have chosen to limit the spectrum of control strategies as much as possible. Consequently, I do not let the worm control the friction but rather use constant asymmetric coefficients (see [Mi188]) whose value is determined according to the sign of the total force acting on each mass. This setting guarantees progress in the preferred direction. I have limited further the range of possibilities by letting the applied force assume only two values, 0 and some positive constant $F_{0}$, so that at each time step the only decision is whether or not to apply force. An external observer who knows all the parameters of the spring could possibly 
calculate some optimal $F(t)$ but we are interested in control "from the inside" based on quantities measurable by the object itself. It turned out that a first approximation of a good control strategy is: expand when the spring is expanding, i.e., as long as the current length is bigger than the previous one. And by employing this control rule the worm indeed crawls across a wide range of environmental parameters (e.g., spring coefficients, frictions, force magnitudes).

The next steps (see [MADL92]) consist of extending the model along several dimensions. In particular the following models have been constructed and experimented with:

- A two-dimensional model in the plane with the capability to change orientation by applying different magnitude of force in the left and right springs.

- A one-dimensional multi-segment model.

- A two-dimensional model with gravitation.

The choice of a research problem and of its associated formal models are not something that can be formally justified. Yet, my feeling is that the behavior of such mechanisms is a fundamental phenomenon worthy of being investigated. The current approach, although it is somewhat naive ${ }^{7}$ from the control theorist's point of view, will be proved fruitful as we proceed to the control of more complex objects operating in non-uniform environments where the theory of optimal control does not have much to offer. The formal analysis of such mechanisms whose dynamics consists of alternating dicrete-continuous phases requires, in fact, a synthesis of of control and automata-theoretic methods. Such a synthesis is currently carried out under the title of Hybrid Systems (see for example [MMP92]) and it is hoped that such techniques could be used to prove analytically what has been demonstrated using simulations, namely, that a worm, equipped with a given control program always progresses.

\footnotetext{
${ }^{7}$ In fact, a recommendation for a naive treatment of motion control is given in [Bra88], but my naivette has developed independently..
} 


\section{Relevant Work}

Some well-known examples of attempts to model, implement and simulate higher-level (with respect to the worm) creatures are Arbib's computational frog ([Arb82]), Brooks' six-legged robot ([Bro89]) and Beer's simulated insect ([Bee90]). On the other side of the worm, Braitenberg's "vehicles" ([Bra84] are approximate models of tiny creature operating closer to the chemical scale.

An interesting and relevant example of biologically-inspired robotics can be found in the works of Hirose and his collaborators ([HU76, HUO81, HM90]). Influenced by the locomotion of snakes they designed and implemented a novel class of robotic objects, the active cord mechanism, which perform a wide variety of tasks such as traversing a narrow maze, overcoming obstacles and gripping, all based on a uniform segmented structure and relying heavily on tactile sensors. In biology, the use of elastic mechanisms in locomotion has been investigated by Alexander [Ale88] who has recently suggested to apply some of the underlying principles to non-wheeled robotic movement ([Ale90]). In a completely different context, Miller ([Mi188, Mi191]) produced realistic animations of worms and snakes using mechanically-based simulations. The worm model employed by our simulator is similar to his.

A very wide-scope worm modeling project is currently carried out by Rockland ([Roc89]). Motivated by the availability of an enormous amount of detailed biological data concerning the nematodes Caenorhabditis Elegans and Ascaris (e.g, [Ken88], [SDADJ85]), this multi-disciplinary project is meant to develop a conceptual framework as well as software tools for modeling the structure and organization of complex biological systems. This modeling effort is directed toward many biological aspects that I have chosen to ignore. A recent result from this project is the development and analysis of a partial model of a segmental oscillator based on biological data ([RR90]).

As for the material realization of worm-like mechanisms, natural and artificial contractile fibers have been widely investigated. Recently several actuator architectures based on polymer gels have been suggested (e.g, [C89], [Br90]). Currently such contraction mechanisms are activated by electrical pulses or, more often, by chemical means such as Aceton. I believe that if an appropriate solution to the energy supply problem will be found, and if the control principles for this class of mechanisms will be understood, the combination of this technology with VLSI will make artificial worms a reality. 


\section{References}

[Ale88] R.M. Alexander, Elastic Mechanisms in Animal Movement, Cambridge University Press, Cambridge, England, 1988.

[Ale90] R.M. Alexander, Three Uses of Springs on Legged Locomotion, The International Journal of Robotics Research, 9, 53-61, 1990.

[Arb82] M.A. Arbib, Rana Computatrix: an Evolving Model of VisuoCoordination in Frog and Toad, in: J.E. Hayes, D. Michie and Y-H. Pao (Eds.), Machine Intelligence 10, Ellis Horwood, Chichester, England, 1982.

[ADH89] B. Arnaldi, G. Dumont, and G. Hégron. Dynamics and Unification of Animation Control, The Visual Computer 5, 22-31, 1989 .

[ADH91] B. Arnaldi, G. Dumont, and G. Hégron, Animation of Physical Systems from Geometric, Kinematic and Dynamic Models, Proc. of Modeling in Computer Graphics, 37-53, Springer, Berlin, 1991.

[Bee90] R. Beer, Intelligence as Adaptive Behavior: an Experiment in Computational Neuroethology, Academic Press, New York, 1990.

[Bra84] V. Braitenberg, Vehicles, MIT Press, Cambridge, MA, 1985.

[Bra88] V. Braitenberg, Some Types of Movements, in C.G. Langton (Ed.), Proc. of Artificial Life, Addison-Wesley, 1988.

[Br90] D.L. Brock, Design and Control of an Artificial Muscle, Unpublished manuscript, MIT AI Lab, 1990.

[Bro86] R.A. Brooks, Achieving Artificial Intelligence through Building Robots, AI Memo 899, MIT AI Lab, 1986.

[Bro89] R.A. Brooks, A Robot that Walks: Emergent Behavior from a Carefully Evolved Network, Neural Computations, 1, 253-263, 1989. 
[CT89] D.G. Caldwell and P.M. Taylor, An Artificial Muscle Actuator for Robots, in K.J. Waldron (Ed.), Advanced Robotics: 1989, 244-258, Springer, Berlin, 1989.

[FG76] V. Fretter and A. Graham, A Functional Anatomy of Invertebrates, Academic Press, New York, 1976.

[HU76] S. Hirose and Y. Umetani, Kinematic Control of Active Cord Mechanism with Tactile Sensors, Proc. of the 2nd ROMANSY Symp., 241-252, Elsevier, Amsterdam, 1976.

[HUO81] S. Hirose, Y. Umetani and S. Oda, An Active Cord Mechanism with Oblique Swivel Joints and its Control, Proc. of the 4 th ROMANSY Symp., 327-340, PWN, Warsaw, 1981.

[HM90] S. Hirose and A. Morishima, Design and Control of a Mobile Robot with an Articulated Body, Int. J. of Robotics Research, 9, 99-114, 1990.

[Ken88] C. Kenyon, The Nematode Caenorhabditis Elegans, Science, 240, 1448-1453, 1988.

[MAD91] O. Maler, B. Arnaldi and G. Dumont, Simulation Experiments with an Artificial Worm, in: G. Hégron (Ed.),Proc. 2nd Eurographics Workshop on Animation and Simulation, 215-221, 1991.

[MADL92] O. Maler, B. Arnaldi, G. Dumont and C. Lecerf, Simulation Experiments with a Single-segment Artificial Worm on a Twodimensional Surface without Gravity, to appear as IRISA technical report.

[MMP92] O. Maler, Z. Manna, and A. Pnueli. From timed to hybrid systems, in: J. de Bakker, C. Huizing, W. de Roever, and G. Rozenberg, eds., Proceedings of the REX Workshop "Real-Time: Theory in Practice", Lect. Notes in Comp. Sci., Vol. 600 (Springer, Berlin, 1992) 28-44.

[MG90] J.-A. Meyer and A. Guillot, Simulation of Adaptive Behavior in Animats: Review and Prospect, in J.-A. Meyer and S.W. Wilson 
(Eds.), From Animals to Animats, Proc. of the First Conf. on Simulation of Adaptive Behavior, Paris, 1990.

[Mi188] G.S.P. Miller, The Motion Dynamics of Snakes and Worms, Computer Graphics, 22, 169-173, 1988.

[Mi191] G.S.P. Miller, Goal-directed Animation of Tabular Articulated Figures or How Snakes Play Golf, in: N.I. Badler, B.A. Barsky and D. Zeltzer (Eds.), Making them Move-Mechanics, Control, and Animation of Articulated Figures, Morgan Kaufmann, San Mateo, CA, 1991.

[Roc89] C. Rockland, The Nematode as a Model Complex System: a Program of Research, Working paper 1865, LIDS MIT, 1989.

[RR90] C. Rockland and S. Rowley, Simulation and Analysis of Segmental Oscillator Models for Nematode Locomotion, Report CICSP-212, LIDS MIT, 1990.

[Ros85] S.J. Rosenchein, Formal Theories of Knowledge in AI and Robotics, New Generation Computing, 3, 345-358, 1985.

[Ste82] R.B. Stein, What Muscle Variable(s) does the Nervous System Control in Limb Movement?, Behavioral and Brain Sciences, 5, $535-577,1982$.

[SDADJ85] A.O.W. Stretton, R.E. Davis, J.D. Angstadt, J.E. Donmoyer and C.D. Johnson, Neural Control of Behavior in Ascaris, Trends in Neuroscience, 8, 294-300, 1985.

[Wil90] S.W. Wilson, The Animat Path to AI, in J.A. Meyer and S.W. Wilson (Eds.), From Animals to Animats, Proc. of the First Conf. on Simulation of Adaptive Behavior, Paris, 1990. 